Bull. Austral. Math. Soc.

Vol. 40 (1989) [91-95]

\title{
AN EXAMPLE ON COMPOSITE DIFFERENTIABLE FUNCTIONS IN INFINITE DIMENSIONS
}

\author{
J.A. JARAMILLO
}

\begin{abstract}
We present an example showing that a classical result due to Glaeser about the closedness of composition subalgebras of infinitely differentiable functions cannot be extended to the case of weakly uniformly differentiable functions on Banach spaces.
\end{abstract}

Our starting point in this note is the following result, due to Glaeser:

THEOREM. [3]. Let $U$ and $V$ be open subsets of $\mathrm{R}^{n}$ and $\mathrm{R}^{p}$ respectively, where $n \geqslant p$; and let $\varphi: U \rightarrow V$ be a mapping satisfying the following conditions

1. $\varphi$ is real-analytic;

2. the rank of $\varphi$ equals $p$ on an everywhere dense subset of $U$;

3. $\varphi(U)$ is closed in $V$;

4. for each compact subset $K \subset \varphi(U)$, there exists a compact subset $H \subset U$ such that $\varphi(H)=K$.

Then the composition subalgebra $\mathcal{A}_{\varphi}=\left\{f \circ \varphi: f \in C^{\infty}(V)\right\}$ is closed in $C^{\infty}(U)$, when this algebra carries its natural Fréchet topology (convergence on compact subsets of the function and all its deriatives).

In $[\mathbf{5}, \mathrm{p} .194]$ the question is posed as to whether an infinite dimensional analogue of this Theorem can be given, in the setting of weakly uniformly differentiable functions on a Banach space (see definitions below). By means of an example we will show that such an analogous result is not possible, even under seemingly favourable conditions.

Spaces of weakly uniformly differentiable mappings first appeared in connection with some approximation problems in infinite dimensions [2], and they also arose in a natural way in the study of homomorphisms between algebras of differentiable functions in infinite dimensions $([\mathbf{1}, \mathbf{5}])$. These spaces are defined as follows (see $[2]$ or $[\mathbf{5}]$ ).

For real Banach spaces $E$ and $F$, let $C_{w b u}(E, F)$ denote the space of all mappings $f: E \rightarrow F$ such that, for each bounded subset $B \subseteq E$, the restriction $\left.f\right|_{B}: B \rightarrow F$ is uniformly continuous when we consider the weak topology $w=\sigma\left(E, E^{\star}\right)$ on $B$ and the norm on $F$. For each $n \in \mathbb{N}$, let $\mathcal{P}\left({ }^{m} E, F\right)$ be the space of all $m$-homogenous

Received 26 September, 1988

Research partially supported by CAICYT grant $2197 / 83$.

Copyright Clearance Centre, Inc. Serial-fee code: 0004-9729/89 \$A2.00+0.00. 
continuous polynomials from $E$ to $F$, endowed with its usual norm, and define $\mathcal{P}_{w b u}\left({ }^{m} E, F\right)=\mathcal{P}\left({ }^{m} E, F\right) \cap C_{w b u}(E, F)$, which is a Banach subspace of $\mathcal{P}\left({ }^{m} E, F\right)$. Then the space $C_{w b u}^{\infty}(E, F)$ of weakly unifornly $C^{\infty}$ mappings from $E$ to $F$ is defined as the set of all infinitely continuously Fréchet differentiable mappings $f: E \rightarrow F$ which satisfy the following conditions

(1) for each $m \in N$ and each $x \in E$, the $m^{\text {th }}$ derivative of $f$ at $x, d^{m} f(x)$, belongs to $\mathcal{P}_{\text {wbu }}\left({ }^{m} E, F\right)$;

(2) for each $m \in N, d^{m} f \in C_{w b u}\left(E, \mathcal{P}_{w b u}\left({ }^{m} E, F\right)\right)$.

We will consider on $C_{w b u}^{\infty}(E, F)$ the Fréchet topology defined by the seminorms $f \rightarrow$ $\sup \left\{\left\|d^{m} f(x)\right\|: x \in B\right\}$, where $B$ is a bounded subset of $E$ and $m \in N$.

We denote $C_{w b u}^{\infty}(E)=C_{w b u}^{\infty}(E, \dot{H})$, which is a Fréchet algebra. The homomorphisms between these algebras were studied in [1] (see also [5]), where the following representation is obtained: Let us consider on the bidual $E^{\star \star}$ the topology $b w^{\star}$, that is, the finest topology which agrees with $w^{\star}=\sigma\left(E^{\star \star}, E^{\star}\right)$ on bounded subsets; $E^{\star \star}$ endowed with the $b w^{\star}$ topology is a locally convex space which will be denoted by $E_{b u}^{\star \star} \star$. For locally convex spaces $X$ and $Y, C^{\infty}(X, Y)$ is the space of all infinitely continuously Fréchet differentiable mappings from $X$ to $Y$ (see $[6,1.8]$; see also $[4,2.9]$, where this is called "bounded differentiability"), and $C^{\infty}(X)$ means $C^{\infty}(X, \mathrm{R})$. Then each function $f \in C_{w b u}^{\infty}(E)$ admits a unique extension $e(f) \in C^{\infty}\left(E_{b w}^{\star \star}\right)$ and, if $E^{\star}$ has the Bounded Approximation Property, then every homomorphism $A: C_{w b u}^{\infty}(E) \rightarrow C_{w b u}^{\infty}(F)$ is induced by a mapping $\varphi \in C^{\infty}\left(F_{b w^{\star}}^{\star \star}, E_{b w^{\star}}^{\star}\right)$, by the formula: $A(f)=\left.e(f) \circ \varphi\right|_{F}$. The question arises of giving conditions analagous to those of Glaeser's Theorem, on the mapping $\varphi$, for the range of the corresponding induced homomorphism to be closed; in this context the following example is interesting:

Example. Let $E$ be one of the spaces $c_{0}$ or $\ell_{p}(1<p<\infty)$. There exists a mapping $\varphi: E \rightarrow E$ satisfying the following conditions

1. $\varphi$ is a (nonhomogeneous) continuous poloynomial of degree $\leqslant 3$;

2. $\varphi: E \rightarrow E$ is a $C^{\infty}$-diffeomorphism;

3. $\varphi$ admits an extension $\bar{\varphi} \in C^{\infty}\left(E_{b w^{\star}}^{\star \star}, E_{b w}^{\star \star}\right)$, and therefore $\varphi$ induces by composition a homomorphism $A: C_{w b u}^{\infty}(E) \rightarrow C_{w b u}^{\infty}(E)$;

4. the extension $\bar{\varphi}: E_{b w}^{\star \star} \star \rightarrow E_{b w}^{\star \star} \star$ is a homeomorphism;

5. the composition subalgebra $\mathcal{A}_{\varphi}=\left\{f \circ \varphi: f \in C_{w b u}^{\infty}(E)\right\}$ is dense but not closed in $C_{w b u}^{\infty}(E)$.

Proof:

1. Let us cousider the sequence of polynomials in one variable

$$
\varphi_{n}(t)=t^{3}-3 t^{2}+\left(3+n^{-3}\right) t ; \quad(t \in \mathbf{R}, \quad n \in \mathbb{N})
$$


It is clear that each $\varphi_{\mathbf{n}}: \mathbf{R} \rightarrow \mathbf{R}$ is a diffeomorphism, and it is not difficult to check that a given sequence $\left(x_{n}\right)_{n \in N}$ belongs to $E$ if, and only if, $\left(\varphi_{n}\left(x_{n}\right)\right)_{n \in N}$ also belongs to $E$; therefore we can define a bijection $\varphi: E \rightarrow E$ by the formula

$$
\varphi\left(\left(x_{n}\right)_{n \in N}\right)=\left(\varphi_{n}\left(x_{n}\right)\right)_{n \in N}
$$

Now $\varphi=P_{3}+P_{2}+P_{1}$, where $P_{1}\left(\left(x_{n}\right)_{n \in N}\right)=\left(\left(3+n^{-3}\right) x_{n}\right)_{n \in N}, P_{2}\left(\left(x_{n}\right)_{n \in N}\right)=$ $\left(-3 x_{n}^{2}\right)_{n \in N}$ and $P_{3}\left(\left(x_{n}\right)_{n \in N}\right)=\left(x_{n}^{3}\right)_{n \in N} ;$ since.each $P_{m} \in \mathcal{P}\left({ }^{m} E, E\right)$ for $m=1,2,3$, we have that $\varphi$ is a continuous polynomial of degree $\leqslant 3$; In particular, $\varphi \in C^{\infty}(E, E)$.

2. Given $x=\left(x_{n}\right)_{n \in N}, y=\left(y_{n}\right)_{n \in N} \in E$, we have that

$$
d \varphi(x)(y) \mp\left(\varphi_{n}^{\prime}\left(x_{n}\right) \cdot y_{n}\right)_{n \in N} .
$$

Since the sequence $\left(\left(\varphi_{n}^{\prime}\left(x_{n}\right)\right)^{-1}\right)_{n \in N}$ is bounded, it follows that $d \varphi(x): E \rightarrow E$ is a topological isomorphism. Then from the Inverse Function Theorem we obtain that $\varphi: E \rightarrow E$ is a $C^{\infty}$-diffeomorphism.

3. It is shown in $[5,11.2]$ that a mapping $\varphi \in C^{\infty}(E, E)$ admits an extension $\bar{\varphi} \in C^{\infty}\left(E_{b w^{\star}}^{\star \star}, E_{b w^{\star}}^{\star \star}\right)$ if and only if $\eta \circ \varphi \in C_{w b u}^{\infty}(E)$ for each $\eta \in E^{\star}$. Then we set $\varphi=P_{3}+P_{2}+P_{1}$ as before, and it will be sufficient to show that $\eta \circ P_{m} \in C_{w b u}(E)$, for each $\eta \in E^{\star}$ and $m=1,2,3$.

Let us consider the case $E=\ell_{p}(1<p<\infty)$. Given $\eta=\left(\eta_{n}\right)_{n \in N} \in \ell_{q}$ (where $p^{-1}+q^{-1}=1$ ), $\varepsilon>0$, and an $R$-ball centred at $0, B_{R} \subseteq E$, we take $K=\sup \left\{\left\|P_{m}(x)\right\|_{p}: x \in B_{R}, \quad m=1,2,3\right\}$ and we choose $N \in N$ such that

$$
\sum_{n>N}\left|\eta_{n}\right|^{q} \leqslant(\varepsilon / K)^{q}
$$

Now let us write $P_{m}\left(\left(x_{n}\right)_{n \in N}\right)=\left(P_{m}^{n}\left(x_{n}\right)\right)_{n \in N} ;$ using the uniform continuity on $[-R, R]$ of $P_{m}^{n}(n=1, \ldots, N ; m=1,2,3)$, we can find some $\delta>0$ such that, if $x=\left(x_{n}\right)_{n \in N}, y=\left(y_{n}\right)_{n \in N} \in B_{R}$ and $\left|x_{n}-y_{n}\right|<\delta$ for $n=1, \ldots, N$, then

$$
\begin{aligned}
\left|\eta \circ P_{m}(x)-\eta \circ P_{m}(y)\right| & \leqslant \sum_{n=1}^{N}\left|\eta_{n}\right| \cdot\left|P_{m}^{n}\left(x_{n}\right)-P_{m}^{n}\left(y_{n}\right)\right|+\sum_{n>N}\left|\eta_{n}\right| \cdot\left|P_{m}^{n}\left(x_{n}\right)-P_{m}^{n}\left(y_{n}\right)\right| \\
& \leqslant \varepsilon+\left(\sum_{n>N}\left|\eta_{n}\right|^{q}\right)^{1 / q} \cdot\left(\sum_{n>N}\left|P_{m}^{n}\left(x_{n}\right)-P_{m}^{n}\left(y_{n}\right)\right|^{p}\right)^{1 / p}<3 \varepsilon
\end{aligned}
$$

The case $E=c_{0}$ is analogous.

4. We start with the case $E=c_{0}$. It is not difficult to check, by the uniqueness, that the extension $\bar{\varphi}: \ell_{\infty} \rightarrow \ell_{\infty}$ of $\varphi$ is defined by the same formula

$$
\bar{\varphi}\left(\left(x_{n}\right)_{n \in \mathbb{N}}\right)=\left(\varphi_{n}\left(x_{n}\right)\right)_{n \in \mathbb{N}} .
$$


Therefore $\bar{\varphi}:\left(\ell_{\infty}, b w^{\star}\right) \rightarrow\left(\ell_{\infty}, b w^{\star}\right)$ is a continuous bijection. In order to see that $\bar{\varphi}$ is a homeomorphism, it is sufficient to show that $\bar{\varphi}^{-1}(B) \subset \ell_{\infty}$ is bounded, for each bounded subset $B \subset \ell_{\infty}$; and this follows immediately from the fact that each inverse function $\varphi_{n}^{-1}: R \rightarrow R$ is increasing.

In the case $E=\ell_{p}(1<p<\infty)$ we have that $\bar{\varphi}=\varphi$ and it is also sufficient to show that $\varphi^{-1}(B) \subset \ell_{p}$ is bounded, for each bounded subset $B \subset \ell_{p}$. Now let us consider the $R$-ball centred at $0, B_{R} \subset \ell_{p}$. We know that if $|t| \leqslant 7 / 8$, then $\left|\varphi_{n}^{-1}(t)\right| \leqslant 1 / 2$ for each $n \in N$; let $n_{R} \in N$ be such that for each $\left(y_{n}\right)_{n \in N} \in B_{R}$, $\operatorname{card}\left\{n \in \mathrm{N}:\left|y_{n}\right| \geqslant 7 / 8\right\} \leqslant n_{R}$, and take $M=\sup \left\{\left|\varphi_{n}^{\prime}(s)\right|^{-1}: n \in N,|s| \leqslant 1 / 2\right\} ;$ note that $M<\infty$. Given $y=\left(y_{n}\right)_{n \in N} \in B_{R}$ we have that, if $\left|y_{n}\right| \leqslant 7 / 8$, by the Mean Value Theorem $\left|\varphi_{n}^{-1}\left(y_{n}\right)\right| \leqslant\left|\left(\varphi_{n}^{-1}\right)^{\prime}\left(\alpha_{n}\right)\right| \cdot\left|y_{n}\right|$ for some $\alpha_{n} \in\left[0, y_{n}\right]$, and since $\left|\varphi_{n}^{-1}\left(\alpha_{n}\right)\right| \leqslant 1 / 2$ it follows that $\left|\varphi_{n}^{-1}\left(y_{n}\right)\right| \leqslant M \cdot\left|y_{n}\right|$; therefore

$$
\begin{aligned}
\sum_{n=1}^{\infty}\left|\varphi_{n}^{-1}\left(y_{n}\right)\right|^{p} & \leqslant \sum_{\left|y_{n}\right|>\tau / 8}\left|\varphi_{n}^{-1}\left(y_{n}\right)\right|^{p}+\sum_{\left|y_{n}\right| \leqslant \tau / 8}\left|\varphi_{n}^{-1}\left(y_{n}\right)\right|^{p} \\
& \leqslant n_{R} \cdot M_{R}^{p}+M^{p} \cdot \sum_{n=1}^{\infty}\left|y_{n}\right|^{p} \\
& \leqslant n_{R} \cdot M_{R}^{p}+M^{p} \cdot R^{p}
\end{aligned}
$$

where $M_{R}=\sup \left\{\left|\varphi_{n}^{-1}(R)\right|, \quad\left|\varphi_{n}^{-1}(-R)\right|: n \in \mathbb{N}\right\}$.

5. Let us consider the space $\mathcal{P}_{f}(E)$ of all continuous real polynomials on $E$ of finite type; that is, the space generated by the functions $\varphi^{m}$, where $\varphi \in E^{\star}$ and $m \in N$ (here $\left.\varphi^{m}(x)=(\varphi(x))^{m}\right)$. To see that $\mathcal{A}_{\varphi}=\left\{f \circ \varphi: f \in C_{w b u}^{\infty}(E)\right\}$ is dense in $C_{w b u}^{\infty}(E)$, we will use the fact that $\mathcal{P}_{f}(E)$ is dense in $C_{w b u}^{\infty}(E)$ and this space induces on each $\mathcal{P}\left({ }^{\prime \prime} E\right) \cap \mathcal{P}_{f}(E)(m \in N)$ the norm topology (see [2] or [5]).

Suppose $E=c_{0}$. Now given $\psi=(\psi)_{n \in \mathbb{N}} \in E^{\star}=\ell_{1}, m \in N$ and $0<\varepsilon<1$, we choose $N \in N$ such that $\sum_{n>N}\left|\psi_{n}\right|<\varepsilon$; let us denote $\eta=\psi^{m}$ and let us consider $\bar{\eta} \in \mathcal{P}_{f}(E)$ defined by $\bar{\eta}\left(\left(x_{n}\right)_{n \in N}\right)=\left(\sum_{n=1}^{N} \psi_{n} \cdot x_{n}\right)^{m}$. Then $f=\bar{\eta} \circ \varphi^{-1} \in C_{w b u}^{\infty}(E)$ and we have that, in the norm of $\mathcal{P}\left({ }^{n} E\right)$,

$$
\|f . \circ \varphi-\eta\|=\|\bar{\eta}-\eta\| \leqslant\left(\sum_{i=1}^{m-1}\|\psi\|^{i} \cdot(1+\|\psi\|)^{m-1}\right) \cdot \varepsilon .
$$

The case $E=\ell_{p}$ is analogous.

Next we will show that $\mathcal{A}_{\varphi} \neq C_{w b u}^{\infty}(E)$. First notice that for each $f \in C_{w b u}^{\infty}(E)$ and the standard basis $\left(e_{n}\right)_{n \in N}$ of $E$,

$$
d(f \circ \varphi)\left(e_{n}\right)\left(e_{n}\right)=d f\left(\varphi\left(e_{n}\right)\right)\left(d \varphi\left(e_{n}\right)\left(e_{n}\right)\right)=n^{-3} d f\left(a_{n}\right)\left(e_{n}\right),
$$


where $a_{n}=\varphi\left(e_{n}\right)$; since $d f: E \rightarrow E^{\star}$ is weakly uniformly continuous on each bounded subset of $E$ and $\left(a_{n}\right)_{n \in N}$ is weakly convergent to 0 , it follows that $\left(\left\|d f\left(a_{n}\right)-d f(0)\right\|\right)_{n \in N}$ is convergent to 0 and $\left(\left\|d f\left(a_{n}\right)\right\|\right)_{n \in N}$ is bounded; thus for each $h=f \circ \varphi \in \mathcal{A}_{\varphi}$ we have that

$$
\left|n^{2} d h\left(e_{n}\right)\left(e_{n}\right)\right|=n^{-1}\left|d f\left(a_{n}\right)\left(e_{n}\right)\right| \leqslant n^{-1}\left\|d f\left(a_{n}\right)\right\|,
$$

and this sequence is convergent to 0 . Nevertheless, let us consider $\psi\left(n^{-2}\right)_{n \in N} \in E^{\star} \subset$ $C_{w b u}^{\infty}(E)$; here $d \psi(x)=\psi$ for each $x \in E$ and $n^{2} d \psi\left(e_{n}\right)\left(e_{n}\right)=1$; this shows that $\psi \notin \mathcal{A}_{\varphi}$.

\section{REFERENCES}

[1] R. Aron, J. Gómez and J. Llavona, 'Homomorphisms between algebras of differentiable functions in infinite dimensions', Mich. Math. J. 35 (1988), 163-178.

[2] R. Aron and J. Prolla, 'Polynomial approximations of differentiable functions on Banach spaces', J. Reine Angew. Math. 313 (1980), 195-216.

[3] G. Glaeser, 'Fonctions composées différentiables', Ann. of Math. 77 (1963), 193-209.

[4] H.H. Keller, Differential Calculus in Locally Convex Spaces, Lecture Notes in Math 417 (SpringerVerlag, Berlin, Heidelberg, New York, Tokyo, 1974).

[6] J. Llavona, Approximation of Continuously Differentiable Functions, North Holland Math. Studies $130,1986$.

[6] S. Yamamuro, Differential Calculus in Topological Linear Spaces, Lecture Notes in Math. 374 (Springer-Verlag, Berlin, Heidelberg, New York, Tokyo, 1974).

Departmento de Análisis Matemático, Universidad Complutense, 28040 Madrid;

Spain. 